\title{
The long non-coding RNA AK023948 enhances tumor progression in hepatocellular carcinoma
}

\author{
BAILIANG YE, BINGREN HU, ZHIHAI ZHENG, RU ZHENG and YIXIONG SHI
}

Department of Laparoscopic Surgery, The First Affiliated Hospital of

Wenzhou Medical University, Wenzhou, Zhejiang 325000, P.R. China

Received December 29, 2016; Accepted May 16, 2017

DOI: $10.3892 /$ etm.2017.5019

\begin{abstract}
The long non-coding RNAs (lncRNAs) have been demonstrated to play pivotal roles in a broad range of processes including tumor biology. However, the exact contributions of lncRNAs to hepatocellular carcinoma (HCC) remain poorly defined. In current study, we have unraveled a novel function of AK023948 in HCC. We found that AK023948 was substantially upregulated in tumor tissues. Meanwhile, higher AK023948 expression correlated with poor survival. Upregulation of AK023948 expression can promote HepG2 and Hep3B proliferation and invasion in in vitro experiments. Furthermore, AK023948 also decreased tumor growth in vivo. The tumorigenic role of AK023948 was partially ascribed to PI3K/Akt/mTOR signaling and AK023948 knockdown decreased pathway activation and tumor growth. These data collectively suggest an oncogenic role for AK023948 and may provide potential insight into therapeutic intervention.
\end{abstract}

\section{Introduction}

The hepatocellular carcinoma (HCC) is among the most common tumors worldwide with increasing occurrence especially in China (1). Despite numerous efforts have been made in comprehensive treatment, the prognosis for HCC patients remain poor possibly owing to late diagnosis, frequent metastasis and lack of efficient therapeutics for cancer with terminal stages. Previous reports have demonstrated myriads of HCC-associated genes and signaling pathways (2-6), but the exact molecular mechanisms responsible for HCC development are still elusive. Therefore, it is in desperate need to identify novel and reliable markers for HCC leading to effectively early diagnosis and intervention.

The long non-coding RNAs (lncRNAs) belong to a class of ncRNAs with over 200 nucleotides in length $(7,8)$. The

Correspondence to: Dr Bingren Hu, Department of Laparoscopic Surgery, The First Affiliated Hospital of Wenzhou Medical University, 2 Fuxue Lane, Wenzhou, Zhejiang 325000, P.R. China

E-mail: hubingren_wmu@163.com

Key words: AK023948, HCC, PI3K/Akt/mTOR, lncRNA
lncRNAs may participate in various biological processes including differentiation, angiogenesis, immune responses and apoptosis $(9,10)$. Recent studies have suggested that the lncRNAs could act either as an oncogene or tumor suppressor with significant contributions to tumorigenesis of many cancers (11-15). For instances, lnc-DILC can inhibit liver cancer progression by modulating IL-6/STAT3 signaling (16). Another ncRNA SBF-AS1 has been reported to play an oncogenic role in non-small cell lung cancer via unknown mechanisms (17). Zhu et al recently identified a 24-lncRNA signature leading to better prognostic value in gastric cancer (18). The metastasis associated in lung adenocarcinoma transcript 1 (MALAT1) can interact with estrogen receptor 1 and result in poor prognosis (19). The lnc-CCAT2 can instead affect energy metabolism through binding to Cleavage Factor I (CFIm) complex to regulate the alternative splicing of Glutaminase (GLS) (20). The serum IncRNA-AF085935 was identified as a potential biomarker for discriminating HCC patients from HBV infection and healthy individuals (21). Therefore, distinct lncRNAs may play differential roles and effectively participate in tumor development.

Although much information has been obtained about the functional diversity of lncRNAs, whether lnc-AK023948 can play a role in tumor progression especially in HCC remains elusive. Here we report a tumorigenic role for lnc-AK023948 in HCC. We found that AK023948 is frequently upregulated in HCC tissues compared with normal adjacent samples. Meanwhile, higher AK023948 expression predicts poor survival. Upregulation of AK023948 expression can promote HepG2 and Hep3B proliferation and invasion while AK023948 knockdown displayed opposite effects. In addition, we argued that AK023948 may elicit these effects via PI3K/Akt pathway. Collectively, these results have provided first insight into the oncogenic role for AK023948 and may potential help diagnosis for HCC.

\section{Materials and methods}

Cell culture and human samples. Five HCC cell lines (HepG2, Hep3B, SNU182, SNU449, MHCC-97H) and CL-48 normal hepatocytes all were purchased from Shanghai Institute of Cell Biology (Shanghai, China) and cultured in RMPI-1640 medium with $3 \%$ fetal bovine serum (FBS; Tiangen, Shanghai, China) plus $100 \mathrm{U} / \mathrm{ml}$ penicillin and streptomycin 
$(100 \mu \mathrm{g} / \mathrm{ml})$ purchased from Tiangen. The surgically sectioned HCC specimens were acquired from The First Affiliated Hospital of Wenzhou Medical University (Wenzhou, China) from July 2010 to October 2014. Immediately after surgical resection, these tissues were stored at $-80^{\circ} \mathrm{C}$ until usage. None patients received preoperative chemotherapy or radiotherapy. All patients have signed formal consent forms. The research for human samples was approved by Ethics Committee of The First Affiliated Hospital of Wenzhou Medical University.

RNA extraction and quantitative PCR. Total RNAs were isolated from both HCC cell lines (HepG2 and Hep3B) and human samples with TRIzol reagent (Invitrogen Life Technologies, Carlsbad, CA, USA) according to the manufacturer's protocol. The cDNA was synthesized from $2 \mathrm{ng}$ of total RNAs with a Reverse Transcription Toolkit (Promega Corp., Madison, WI, USA). The total reaction volume was $20 \mu \mathrm{l}$ and the mixture was prepared as previously described (22). The quantitative PCR requires initial denaturation for $10 \mathrm{~min}$ at $95^{\circ} \mathrm{C}$ and total 35 cycles with final extension at $72^{\circ} \mathrm{C}$ for $5 \mathrm{~min}$. $\triangle C t$ values were normalized to $G A P D H$. Reactions were performed by the ABI PRISM ${ }^{\circledR} 7000$ Sequence Detection System (Applied Biosystem, Foster City, CA, USA) according to the manufacturer's instructions. The relative expression was calculated using the $2^{-\Delta \mathrm{Ct}}$ method $\left(\triangle C t=\mathrm{CT}_{\mathrm{AK} 023948}-\mathrm{CT}_{G A P D H}\right)$. The primer sequences were provided below: $G A P D H$ : forward: 5'-ACTGTGCCGACT TGACGTTT-3'. reverse: 5'-ATC GTAGTGAACGGTCGATTGT-3'. AK023948: forward: 5'-ACCCAGATTCGACGACCTT-3'. reverse: 5'-GCCGGA TTATTACGT-3'.

Microarray and data analysis. We used the Affymetrix Gene Chip miRNA array to identify the potential function of lncRNAs in HCC. Among all patients, three were used for microarray analysis. In short, $200 \mathrm{ng}$ total RNAs were tagged with poly-A tail and the ligation was performed using FlashTag Biotin HSR toolkit before the hybridization following the manufacturer's instructions. The data were scanned by Affymetrix GeneChip Scanner 3000 system and images were converted into values using imbedded programs (23). The robust multichip average (RMA) protocol was used to analyze the data.

Small interfering RNA. The small interfering RNA experiment and negative control (NC) was synthesized and commercially obtained from Tiangen. The target sequences for si \#1 (UUG UGCCCAAUGCAU), si \#2 (UCGAGACACCGUC), si \#3 (UGAGGAUUCCGCUU) were provided. Random scramble controls were also purchased from Tiangen. The transfection was performed using Lipofectamine ${ }^{\circledR} 2000$ (Invitrogen Life Technologies, Shanghai, China). At $36 \mathrm{~h}$ after incubation, the cells were harvested and subject to qPCR or western blotting analyses. Three independent experiments were performed.

Proliferation assay. To quantify proliferation, the Cell Counting Kit-8 (CCK-8; Dojindo, Kumamoto, Japan) was used. After treatment for $24 \mathrm{~h}$, HepG2 and Hep3B cells were re-suspended and seeded into a 12 -well plate $\left(10^{6}\right.$ cells per well) for totally 5 days. A total $10 \mu \mathrm{l}$ MTT solutions were added into the culture with a final concentration of $10 \mathrm{mg} / \mathrm{ml}$.
The crystalline formazan was resolved in $100 \mu \mathrm{l}$ sodium dodecyl sulfate (SDS, 5\%) solution for $24 \mathrm{~h}$. Optical density was evaluated at $490 \mathrm{~nm}$.

Invasion assays. A 12-well transwell plate (Tiangen) was used to measure the invasion. Briefly, $10^{5}$ transfected cells were suspended in serum-free medium and then plated into upper chambers. Then, the upper chamber was coated with Matrigel (Tiangen) overnight. The lower chamber was covered with RPMI-1640 medium and 3\% FBS as chemo-attractants. After $48 \mathrm{~h}$, the cells on the top were removed. Cells into the lower chamber were fixed with $3 \%$ PFA and stained by $0.2 \%$ crystal violet. Leica microscope fluorescent microscope (DM-IRB; Leica, Mannheim, Germany) was used for visualization.

Plasmid construction. The AK023948 was cloned into pcDNA3.1 vector (Invitrogen, Shanghai, China). Transfection with pcDNA-AK023948 was used to overexpress AK023948 in HCC cell lines. An empty pcDNA vector purchased from Invitrogen Life Technologies functioned as a control. The qPCR was used to quantify AK023948 expression. Three independent replicates were implemented.

Western blot analysis. Cells were lysed with RIPA lysis buffer (10 mM Tris- $\mathrm{HCl}, 100 \mathrm{mM} \mathrm{NaCl}, 1 \%$ Triton $\mathrm{X}-100,1 \%$ sodium deoxycholate and $0.1 \%$ SDS). $10 \%$ SDS-polyacrylamide gel electrophoresis (PAGE) was implmented to separate the proteins. We used the polyvinylidene difluoride (PVDF; Tiangen) membranes in current study. Primary antibodies against p85 (a subunit of PI3K), p-p85 (Tyr458), Akt, p-Akt (Ser473), mTOR, p-mTOR (Ser2448) or GAPDH were purchased from Tiangen. After being washed with TBST, the blots were incubated with horseradish peroxidase-conjugated secondary antibodies (Tiangen). Chemi-luminescence was used to visualize blots (GE Healthcare, Fairfield, CT, USA).

Immunohistochemistry and in situ hybridization. We used the immunohistochemistry technique with the Expose HRP/DAB detection system kit (Abcam, Shanghai, China). Briefly, after demasking, the lnc-AK023948 was synthesized to DIG-labeled LNA probes. The digoxigenins was detected by anti-DIG specific antibody with alkaline phosphatase conjugation. The detailed method was performed as previously described (24).

In vivo implantation. Transfection of HepG2 cells was performed using lenti-virus transfection system. The system was initially maintained for $12 \mathrm{~h}$ and then cultured for another $36 \mathrm{~h}$. Then, cells were re-suspended and totally $10^{6}$ cells were injected subcutaneously into the nude mice (Model Animal Research Center, Nanjing, China). At 30 days later, mice were sacrificed by sodium amobarbital overdose $(200 \mathrm{mg} / \mathrm{kg})$ and solid tumors were weighted. Images were shown with $\mathrm{x} 400$ magnification.

Statistical analysis. Results were displayed as mean \pm SD. Statistical significance were determined by Mann-Whitney test (version 16.0; SPSS, Inc., Chicago, IL, USA) andP $<0.05$ was considered to indicate a statistically significant difference. Kaplan-Meier survival curve was tested using log-rank test. Fisher exact test was used to evaluate the correlation between 
Table I. Deregulated lncRNAs using microarray in HCC patients.

\begin{tabular}{llll}
\hline lncRNAs & Log2 fold-change (T/N) & \multicolumn{1}{c}{$\operatorname{lncRNAs}$} & Log2 fold-change (T/N) \\
\hline FJ573250 & -3.74472852806546 & NR_033768 & 3.36551564617163 \\
ENSG00000234741 & -2.40506289289636 & ENSG00000220635 & 3.00086150154706 \\
ENSG00000225783 & -2.54086116273159 & LOC285194 & 2.96770318391678 \\
ENSG00000251562 & -2.00636903699041 & Gm16197 & 2.80417556809907 \\
ENSG00000240990 & -2.35395453692240 & ENSG00000253552 & 3.42310336515152 \\
ENST00000474046 & -2.11767942519340 & HULC & 2.87681190069810 \\
Khps1 & -2.17855339388809 & ENST00000383038 & 2.70780848415775 \\
Msx1as & -1.79269869943132 & 7SL & 3.11109811537829 \\
CASC2 & -1.68281127017120 & Emx2os & 2.67291021808786 \\
NR_026875 & -1.68868907303582 & HOTTIP & 2.68298468553566 \\
ENSG00000240498 & -1.67691344229839 & ZEB1-AS1 & 2.06012365818692 \\
linc1458 & -1.32135340559836 & AK023948 & 3.72871538236571 \\
LUST & -1.29067370181886 & PTENP1 & 2.19828407145188 \\
\hline
\end{tabular}

HCC samples vs. NAT samples; False discovery rate $<0.1 \%, \mathrm{P}<0.01$. IncRNAs, long non-coding RNAs; HCC, hepatocellular carcinoma; NAT, normal adjacent tissue.

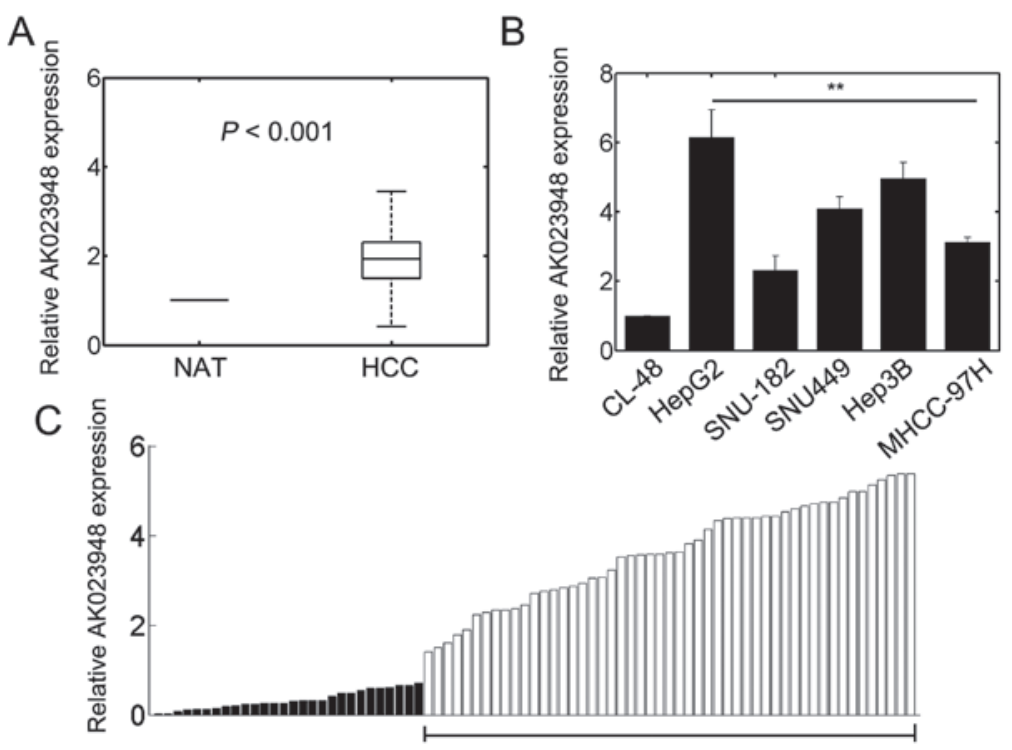

Figure 1. The lncRNA AK023948 was upregulated in hepatocellular carcinoma (HCC). (A) qPCR results for 79 HCC samples as well as corresponding normal adjacent tissues (NATs). (B) Expression of AK023948 in normal cell line CL-48 and 5 HCC cell lines. ${ }^{* *} \mathrm{P}<0.01$. (C) Quantification of AK023948 expression in 79 HCC samples. The cut-off value was determined as indicated. 51 samples were identified as high expression (white bars).

lnc-AK023948 and clinicopathological characteristics. Experiments were performed by at least triplicates.

\section{Results}

AK023948 is upregulated in HCC tissues and cell lines. Based on the lncRNA expression from microarray data (Table I), we determined to investigate the role of AK023948 in HCC. Totally, 79 samples were subject to qRT-PCR and the results were shown. We found that AK023948 level was frequently raised compared with normal adjacent tissues (NATs) (Fig. 1A). Meanwhile, we also checked this tendency in HCC cell lines. We found that qualitatively similar results as AK023948 expression was enhanced in HCC cell lines ( $\mathrm{P}<0.01$, Fig. 1B).
The 79 patients were classified into two separate groups based on the expression AK023948 (Fig. 1C). Then, we evaluated the association of AK023948 with different clinicopathological factors. We noticed that clinicopathological factors expression was not significantly associated with age and gender (Table II). However, AK023948 significantly correlated with TNM stages $(\mathrm{P}=0.004)$, tumor size $(\mathrm{P}<0.0001)$ and metastasis $(\mathrm{P}=0.008)$ (Table II). These results suggested that AK023948 is upregulated in tumor tissues and correlates with malignant features.

High AK023948 expression predicts poor survival. We further examined whether AK023948 levels correlated with patient survival. We obtained the Kaplan-Meier plot and the results 
Table II. The correlation between the lnc-AK023948 and clinicopathological features.

\begin{tabular}{|c|c|c|c|c|}
\hline \multirow[b]{2}{*}{ Clinicopathological parameters } & \multicolumn{3}{|c|}{ lnc-AK023948 expression } & \multirow[b]{2}{*}{$\mathrm{P}$} \\
\hline & Patient no. & Low (n=28) (no., \%) & $\operatorname{High}(\mathrm{n}=51)($ no., $\%)$ & \\
\hline Age & & & & 0.205 \\
\hline$<60$ & 43 & $13(30.2)$ & $30(69.8)$ & \\
\hline$\geq 60$ & 36 & $15(41.7)$ & $21(58.3)$ & \\
\hline Gender & & & & 0.215 \\
\hline Male & 39 & $16(41.0)$ & $23(59.0)$ & \\
\hline Female & 40 & $12(30.0)$ & $28(70.0)$ & \\
\hline TNM stage & & & & $0.004^{\mathrm{a}}$ \\
\hline I-II & 31 & $17(54.8)$ & $14(45.2)$ & \\
\hline III-IV & 48 & $11(22.9)$ & $37(77.1)$ & \\
\hline Tumor size & & & & $<0.0001^{\mathrm{a}}$ \\
\hline$<3 \mathrm{~cm}$ & 30 & $19(63.3)$ & $11(36.7)$ & \\
\hline$\geq 3 \mathrm{~cm}$ & 49 & $9(18.4)$ & $40(81.6)$ & \\
\hline Metastasis & & & & $0.008^{\mathrm{a}}$ \\
\hline Absent & 35 & $18(51.4)$ & $17(48.6)$ & \\
\hline Present & 44 & $10(22.7)$ & $34(77.3)$ & \\
\hline
\end{tabular}

${ }^{\text {aa }}<0.01$.

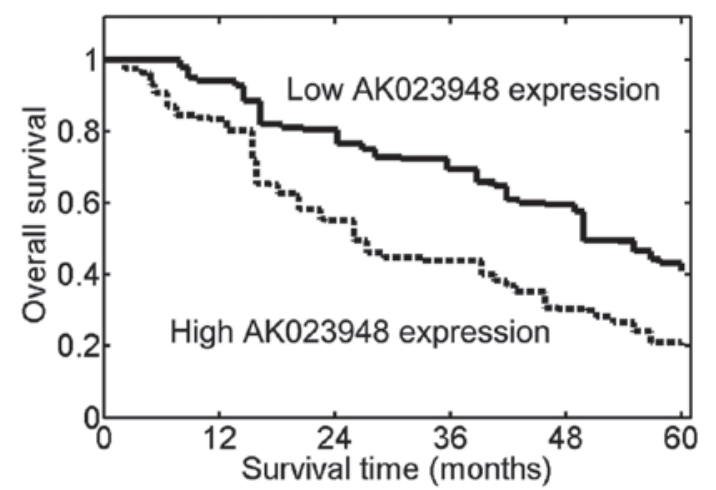

Figure 2. Kaplan-Meier survival curves for hepatocellular carcinoma (HCC) patients. HCC patients with high AK023948 expression had a significantly shorter overall survival $(\mathrm{P}<0.01, \log$-rank test).

showed that higher AK023948 expression lead to poor overall survival ( $\mathrm{P}=0.003$, Fig. 2). The lower branch may reach around $20 \%$ in the 5-year follow-up. These results suggested that high AK023948 level may predict poor survival.

Alterations in AK023948 levels regulate proliferation and invasion. As we have noticed that AK023948 expression is significantly higher in HCC tissues and cell lines compared with normal ones (Fig. 1), we then hypothesized that AK023948 expression may affect oncogenic phenotypes of HCC. We next investigated whether changes in AK023948 levels may influence HCC cell proliferation and invasion. We first synthesized three different siRNA targeting AK023948 and verified the efficiency. We found that all synthetics significantly decreased the intrinsic levels of AK023948 (Fig. 3A). Furthermore, we noticed that \#3 si-RNA was most proficient to knock down AK023948 compared with the other two synthetics (Fig. 3A and B). The knockdown efficiency may be over $80 \%$ for si \#3 (Fig. 3A and B). To overexpress AK023948, we transfected HepG2 and Hep3B cells with pcDNA-AK023948 and quantified the efficiency. The results showed that pcDNA-AK023948 introduction can significantly upregulate AK023948 expression by around 2.5-3-fold (Fig. 3C and D). We further noticed that Hep3B cells were more sensitive to AK023948 overexpression or knockdown (Fig. 3B and D). We then evaluated the effect of AK023948 on the proliferation of HepG2 and Hep3B cells. We found that si-AK023948 mediated knockdown substantially decreased the proliferation of HepG2 and Hep3B cells (Fig. 3E). Consistently, AK023948 overexpression by pcDNA-AK023948 transfection markedly increased the proliferation of HCC cells (Fig. 3E). The anti-proliferation effect became evident as early as the second day but the pro-proliferation effect was significantly only after the third day (Fig. 3E). The invasion assay also displayed qualitatively similar results (Fig. 3F). We found that AK023948 overexpression can promote the invasion of HepG2 and Hep3B cells (Fig. 3F, top and bottom panels). Meanwhile, AK023948 knockdown instead decreased the invasive potential of HepG2 and Hep3B cells (Fig. 3F). These results suggested that AK023948 may promote the proliferation and invasion of HCC cell lines in vitro.

AK023948 may influence PI3K/Akt/mTOR pathways and also exerts oncogenic role in vivo. As PI3K/Akt/mTOR pathways was frequently deregulated in various tumor types (25), we next investigated whether PI3K/Akt/mTOR signaling was altered by modulating AK023948 expression. We found that phosphorylated forms of PI3K, Akt and mTOR were significantly decreased if AK023948 was consistently downregulated (Fig. 4A). We further noticed that the total levels of PI3K, Akt 
A
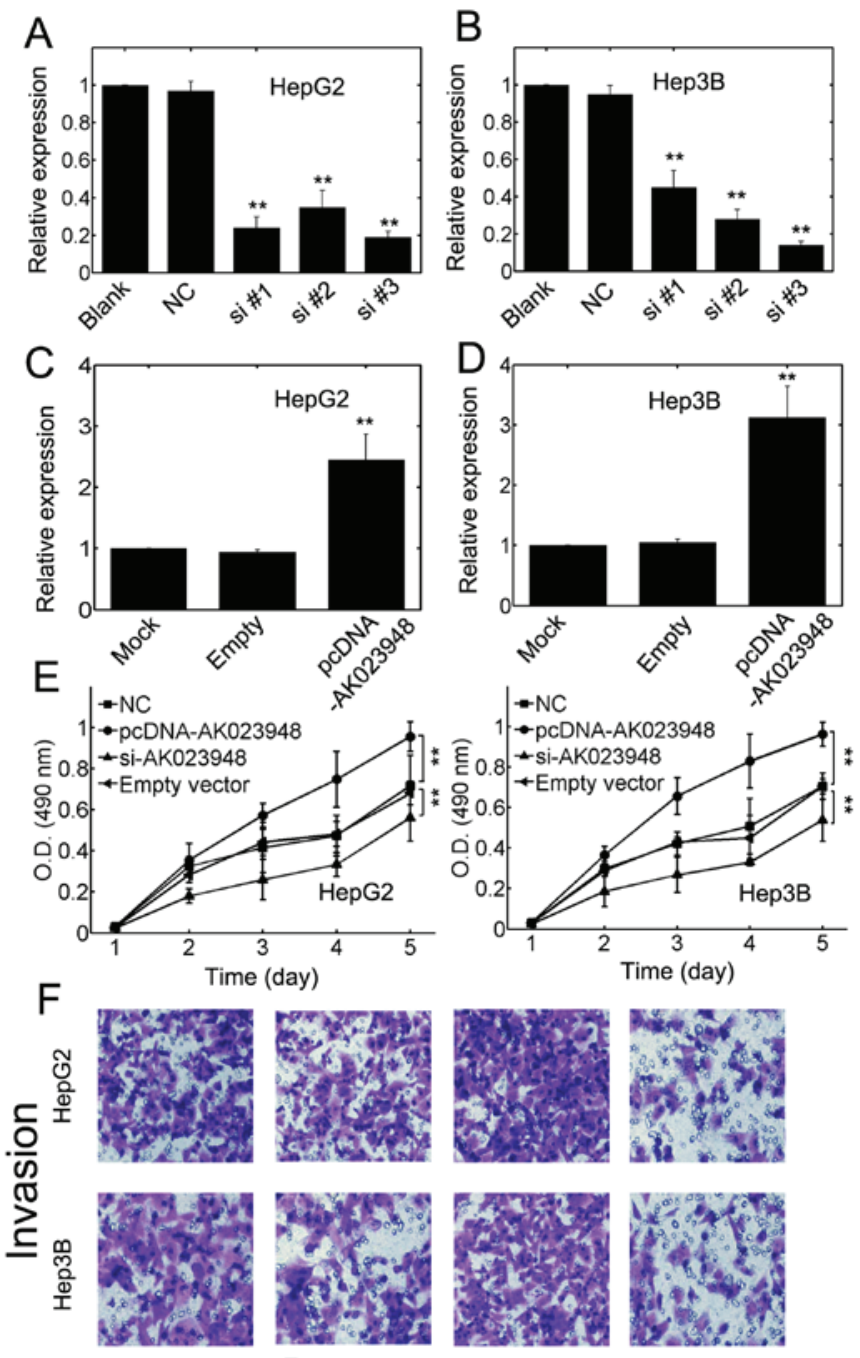

NC

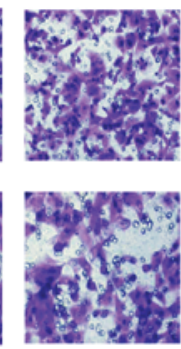

Empty vector

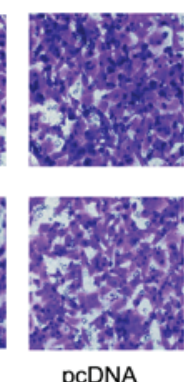

$-A K 023948$

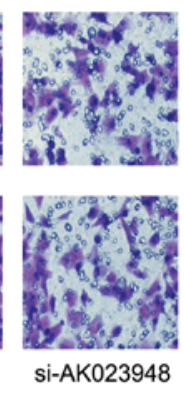

Figure 3. AK023948 can promote hepatocellular carcinoma (HCC) progression. The relative knockdown efficiency for (A) HepG2 and (B) Hep3B cells were shown. The si \#3 showed the highest efficiency. The transfection verification using pcDNA-AK023948 was determined in (C) HepG2 and (D) Hep3B cells. (E) A 5-day proliferation assay for HepG2 (left) and Hep3B (right) cells left untreated or transfected with empty vector, pcDNA-AK023948 or si-AK023948. (F) Invasion assays for HepG2 (top) and Hep3B (bottom) cells were displayed in groups either untreated or transfected with empty vector, pcDNA-AK023948 or si-AK023948.

and mTOR were not obviously changed (Fig. 4A) suggesting that AK023948 primarily regulated activation but not the turnover of PI3K/Akt/mTOR signaling. We also measured the relative activation of these species in 79 samples and the results again confirmed a positive association between AK023948 and phosphorylated PI3K (Fig. 4B). The activated Akt and mTOR also exhibited significantly positive correlation with AK023948 expression (Fig. 4C and D, note that the distribution of AK023948 is equivalent among panels $\mathrm{B}-\mathrm{D})$. To further explore the in vivo effect of AK023948, we injected nude mice with Hep3B cells either untreated or treated with si-AK023948. After 30 days, the solid tumors were evaluated. The in vivo experiments showed that AK023948 knockdown markedly decreased the tumor weight (Fig. 4E). Meanwhile, lowering AK023948 expression also reduced the tumor size accordingly (Fig. 4F). Consistently, HCC tissues showed substantially enhanced AK023948 staining in comparison with NATs (Fig. 4G). These results showed that AK023948 may promote tumor growth in vivo and the effect was partially ascribed to aberrant PI3K/Akt/mTOR activation.

\section{Discussion}

Dysfunction in IncRNA may usually regulate biological systems by affecting epigenetic information and result in preference in cellular proliferation and even uncontrolled tumor development (26). The HCC is characterized by multiple abnormalities and always complicates traditional treatment and intervention (15). In previous reports, lncRNA DANCR can promote stemness features of HCC via regulating CTNNB1 (27). The lnc-ZEB1-AS1 also correlates with HCC metastasis and positively modulates ZEB1 expression (28). Another lnc-AFAP1-AS1 also predicts poor prognosis in HCC through upregulating RhoA/Rac2 signaling (29). Therefore, identifying cancer associated lncRNA may provide critical insight and shed light on potential tumor intervention and diagnosis.

We used microarray assays to identify novel prognostic marker for HCC. We found that AK023948 may potentiate tumor development based on our experimental data. AK023948 is located in TG-SLA (thyroglobulin and Src-like adaptor) locus at chromosome $8 \mathrm{q} 24$, which is a susceptibility locus. It is embedded in the intron of both TG (thyroglobulin, sense orientation) and SLA (Src-like adaptor, antisense) genes. To date, there exist few reports regarding the role of AK023948. By screening, we identified that AK023948 might play a role in HCC.

We further verified the effect of AK023948 in our experiments. We found that AK023948 is upregulated in HCC tissues as well as cell lines. AK023948 also positively correlates with some clinicopathological features such as TNM stages, metastasis and tumor size. Elevated AK023948 expression promotes tumor proliferation and invasion. High AK023948 expression also predicts poor survival within a 5-year follow-up. We further confirmed that AK023948 significantly correlated with PI3K/Akt/mTOR pathway activation and further verified these effects in surgical specimens. In vivo experiments also demonstrated an oncogenic role for AK023948 as AK023948 knockdown can significantly decrease the tumor weight in implantation study. These results collectively demonstrated that AK023948 may potentiate tumor development in HCC.

The PI3K/Akt/mTOR pathway denotes a prominent survival pathway which is aberrantly activated in many cancers. Phosphorylation of Akt has been correlated with poor prognosis in various cancers including liver (30). Amplification, overexpression or mutations in PI3K subunits usually occur in tumors (30). Being a direct downstream target for Akt, mTOR is also over-activated in various tumors and associated with malignant phenotype (31). Numerous mechanisms contribute to the $\mathrm{PI} 3 \mathrm{~K} / \mathrm{Akt} / \mathrm{mTOR}$ pathway activation as reviewed elsewhere (30). Targeting the PI3K/Akt/mTOR pathway in combinatorial therapy may prove to be an effective strategy to cope with cancer. Combining chemo- or radio-therapy with tyrosine kinase inhibitors (TKIs) may become the rationale in conventional treatment. Many clinical trials with Akt pathway inhibitors and other combination agents have been in progress. 

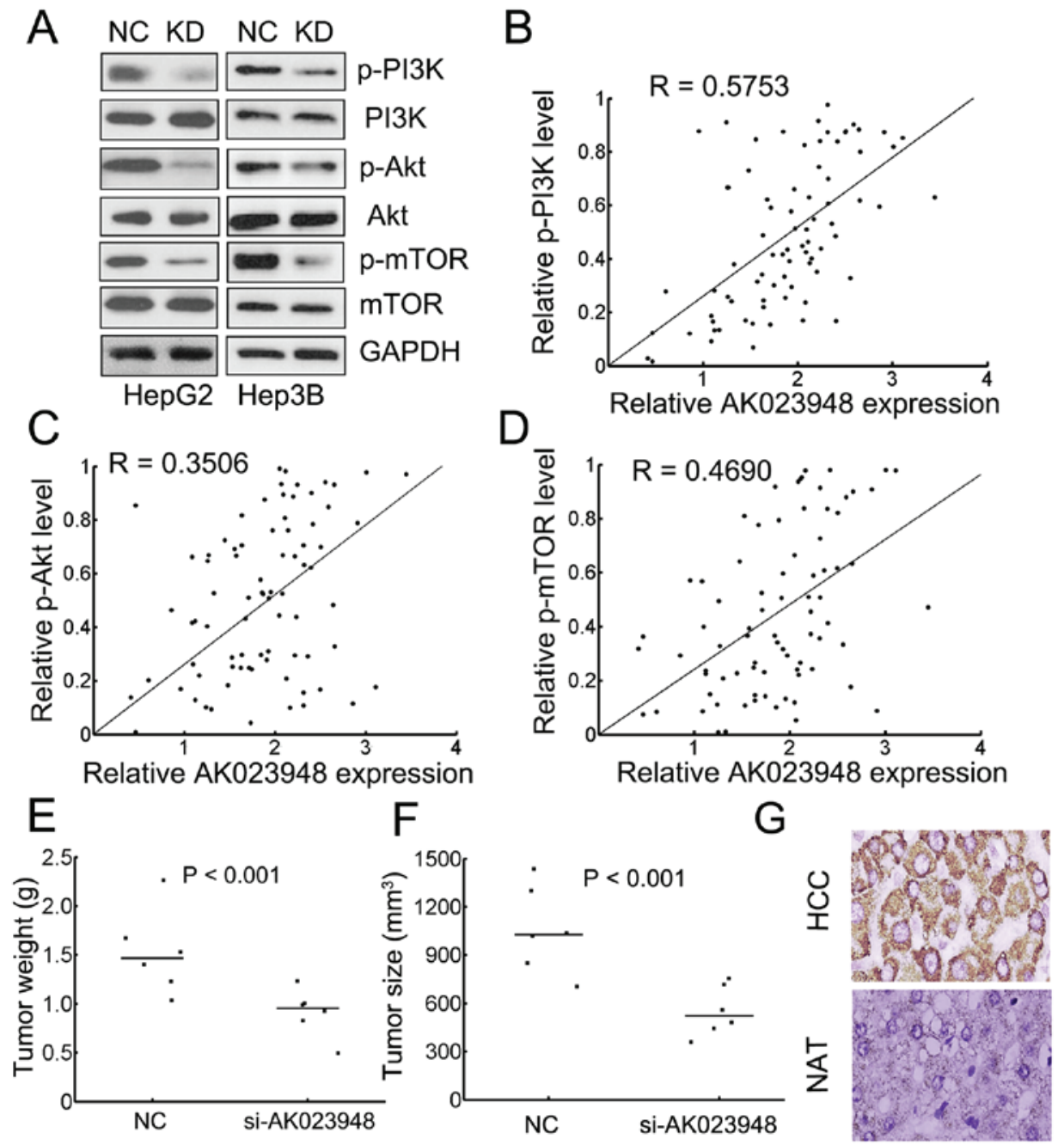

Figure 4. AK023948 can affect PI3K pathway. (A) Western blot for PI3K/Akt/mTOR signaling pathway using specific antibodies. NC, negative control; KD, si-AK023948. The association between relative expression of AK023948 and activated (B) PI3K, (C) Akt and (D) mTOR for 79 specimens were shown. Pearson correlation coefficients were marked. All P-values $<0.0001$. (E) The growth of solid tumors in nude mice injected with HepG2 cells either untreated or transfected with si-AK023948, $\mathrm{n}=6$ for each case. The median levels were indicated. (F) Tumor size after 30 days' implantation, $\mathrm{n}=6$ for each group. The median levels were shown. (G) Immunostaining for intrinsic AK023948 levels in hepatocellular carcinoma (HCC) samples and normal adjacent tissues (NATs).

However, owing to feedback loops especially negative feedback loops in biological signaling pathway and dynamic flux compensation, several combinations of inhibitors may not be effective in reality (32). Therefore, unraveling effective combinatorial agents requires strong concerns in future biological research. In current study, we found that AK023948 inhibitors can markedly downregulate phosphorylated PI3K/Akt/mTOR in western blots (Fig. 4A). AK023948 knockdown can also lead to reduced tumor growth (Table I, Fig. 4E and F). Therefore, strategies targeting AK023948 might be an alternative and efficient way to annihilate HCC development. More efforts should be dedicated to pinpoint this conjecture in future.

In this study, we have for the first time identified a novel role of AK023948 in HCC. Our data revealed that AK023948 may facilitate malignant phenotypes of HCC progression both in vivo and in vitro. Meanwhile, AK023948 positively correlates PI3K/Akt/mTOR pathway activation as AK023948 knockdown significantly downregulate overall phosphorylation. Our data argued that AK023948 could serve as a novel and promising target for therapeutic intervention. With great advance in experimental and computational strategies, detailed molecular mechanisms upon how AK023948 promotes HCC progression can be intricately uncovered.

\section{References}

1. El-Serag HB, Marrero JA, Rudolph L and Reddy KR: Diagnosis and treatment of hepatocellular carcinoma. Gastroenterology 134: 1752-1763, 2008.

2. Cartularo L, Laulicht F, Sun H, Kluz T, Freedman JH and Costa M: Gene expression and pathway analysis of human hepatocellular carcinoma cells treated with cadmium. Toxicol Appl Pharmacol 288: 399-408, 2015.

3. Tuo YL, Li XM and Luo J: Long noncoding RNA UCA1 modulates breast cancer cell growth and apoptosis through decreasing tumor suppressive miR-143. Eur Rev Med Pharmacol Sci 19: 3403-3411, 2015.

4. Yan SR, Liu ZJ, Yu S and Bao YX: Investigation of the value of miR-21 in the diagnosis of early stage HCC and its prognosis: A meta-analysis. Genet Mol Res 14: 11573-11586, 2015.

5. Ma Y, Lu Y and Lu B: MicroRNA and long non-coding RNA in ovarian carcinoma: Translational insights and potential clinical applications. Cancer Invest 34: 465-476, 2016.

6. Sheppard TL: Long non-coding RNAs: Pulsating RNA motifs. Nat Chem Biol 12: 889, 2016.

7. Wang $\mathrm{KC}$ and Chang HY: Molecular mechanisms of long noncoding RNAs. Mol Cell 43: 904-914, 2011. 
8. Jain S, Thakkar N, Chhatai J, Bhadra MP and Bhadra U: Long non-coding RNA: Functional agent for disease traits. RNA Biol: May 26, 2016 (Epub ahead of print).

9. Fatica A and Bozzoni I: Long non-coding RNAs: New players in cell differentiation and development. Nat Rev Genet 15: 7-21, 2014.

10. Maass PG, Luft FC and Bähring S: Long non-coding RNA in health and disease. J Mol Med (Berl) 92: 337-346, 2014.

11. Zhou T, Kim Y and MacLeod AR: Targeting long noncoding RNA with antisense oligonucleotide technology as cancer therapeutics. Methods Mol Biol 1402: 199-213, 2016.

12. Zhao QS, Li L, Zhang L, Meng XW, Li LL, Ge XF and Li ZP Over-expression of lncRNA SBF2-AS1 is associated with advanced tumor progression and poor prognosis in patients with non-small cell lung cancer. Eur Rev Med Pharmacol Sci 20 : 3031-3034, 2016.

13. Sui CJ, Zhou YM, Shen WF, Dai BH, Lu JJ, Zhang MF and Yang JM: Long noncoding RNA GIHCG promotes hepatocellular carcinoma progression through epigenetically regulating miR-200b/a/429. J Mol Med (Berl) 94: 1281-1296, 2016.

14. Lian Y, Cai Z, Gong H, Xue S, Wu D and Wang K: HOTTIP: A critical oncogenic long non-coding RNA in human cancers. Mol Biosyst 12: 3247-3253, 2016.

15. Feo F, Simile MM and Pascale RM: Focal loss of long non-coding RNA-PRAL, as determinant of cell function and phenotype of hepatocellular carcinoma. Ann Transl Med 4: 183, 2016.

16. Wang X, Sun W, Shen W, Xia M, Chen C, Xiang D, Ning B, Cui X, Li H, Li X, et al: Long non-coding RNA DILC regulates liver cancer stem cells via IL-6/STAT3 axis. J Hepatol 64 1283-1294, 2016.

17. Lv J, Qiu M, Xia W, Liu C, Xu Y, Wang J, Leng X, Huang S, Zhu R, Zhao M, et al: High expression of long non-coding RNA SBF2-AS1 promotes proliferation in non-small cell lung cancer. J Exp Clin Cancer Res 35: 75, 2016.

18. Zhu X, Tian X, Yu C, Shen C, Yan T, Hong J, Wang Z, Fang JY and Chen $\mathrm{H}$ : A long non-coding RNA signature to improve prognosis prediction of gastric cancer. Mol Cancer 15: 60, 2016.

19. Huang NS, Chi YY, Xue JY, Liu MY, Huang S, Mo M, Zhou SL and $\mathrm{Wu}$ J: Long non-coding RNA metastasis associated in lung adenocarcinoma transcript 1 (MALAT1) interacts with estrogen receptor and predicted poor survival in breast cancer. Oncotarget 7: 37957-37965, 2016.

20. Redis RS, Vela LE, Lu W, Ferreira de Oliveira J, Ivan C, Rodriguez-Aguayo C, Adamoski D, Pasculli B, Taguchi A, Chen Y, et al: Allele-Specific Reprogramming of Cancer Metabolism by the Long Non-coding RNA CCAT2. Mol Cell 61: $520-534,2016$

21. Lu J, Xie F, Geng L, Shen W, Sui C and Yang J: Investigation of serum lncRNA-uc003wbd and lncRNA-AF085935 expression profile in patients with hepatocellular carcinoma and HBV. Tumour Biol 36: 3231-3236, 2015.
22. Wang J, Cheng G, Li X, Pan Y, Qin C, Yang H, Hua L and Wang Z: Overexpression of long non-coding RNA LOC400891 promotes tumor progression and poor prognosis in prostate cancer. Tumour Biol 37: 9603-9613, 2016.

23. Park JL, Park SM, Kwon OH, Lee HC, Kim JY, Seok HH, Lee WS, Lee SH, Kim YS, Woo KM and Kim SY: Microarray screening and qRT-PCR evaluation of microRNA markers for forensic body fluid identification. Electrophoresis 35: 3062-3068, 2014.

24. Lin Z, Sun L, Chen W, Liu B, Wang Y, Fan S, Li Y and Li J: miR-639 regulates transforming growth factor beta-induced epithelial-mesenchymal transition in human tongue cancer cells by targeting FOXC1. Cancer Sci 105: 1288-1298, 2014.

25. Missiaglia E, Dalai I, Barbi S, Beghelli S, Falconi M, della Peruta M, Piemonti L, Capurso G, Di Florio A, delle Fave G, et al: Pancreatic endocrine tumors: Expression profiling evidences a role for AKT-mTOR pathway. J Clin Oncol 28: 245-255, 2010.

26. Cao L, Zhang Q, Cheng S, Chen Z, Hua Z, Yang J, Liu D and Cui N: Long non-coding RNAs and genes contributing to the generation of cancer stem cells in hepatocellular carcinoma identified by RNA sequencing analysis. Oncol Rep 36: 2619-2624, 2016.

27. Yuan SX, Wang J, Yang F, Tao QF, Zhang J, Wang LL, Yang Y, Liu H, Wang ZG, Xu QG, et al: Long noncoding RNA DANCR increases stemness features of hepatocellular carcinoma by derepression of CTNNB1. Hepatology 63: 499-511, 2016.

28. Li T, Xie J, Shen C, Cheng D, Shi Y, Wu Z, Deng X, Chen H, Shen B, Peng C, et al: Upregulation of long noncoding RNA ZEB1-AS1 promotes tumor metastasis and predicts poor prognosis in hepatocellular carcinoma. Oncogene 35: 1575-1584, 2016.

29. Zhang JY, Weng MZ, Song FB, Xu YG, Liu Q, Wu JY, Qin J, Jin T and Xu JM: Long noncoding RNA AFAP1-AS1 indicates a poor prognosis of hepatocellular carcinoma and promotes cell proliferation and invasion via upregulation of the RhoA/Rac2 signaling. Int J Oncol 48: 1590-1598, 2016.

30. LoPiccolo J, Blumenthal GM, Bernstein WB and Dennis PA: Targeting the PI3K/Akt/mTOR pathway: Effective combinations and clinical considerations. Drug Resist Updat 11: 32-50, 2008.

31. Petroulakis E, Mamane Y, Le Bacquer O, Shahbazian D and Sonenberg N: mTOR signaling: Implications for cancer and anticancer therapy. Br J Cancer 96 (Suppl): R11-R15, 2007.

32. Nguyen LK and Kholodenko BN: Feedback regulation in cell signalling: Lessons for cancer therapeutics. Semin Cell Dev Biol 50: 85-94, 2016. 